\title{
Heterogeneous growth of single crystals on polycrystals
}

\author{
Zumin Wang, ${ }^{1,2, *}$ Lars P. H. Jeurgens, ${ }^{3}$ Lin Gu, ${ }^{4}$ and Eric J. Mittemeijer ${ }^{2,5}$ \\ ${ }^{1}$ School of Materials Science and Engineering, Tianjin University, 300350 Tianjin, China \\ ${ }^{2}$ Max Planck Institute for Intelligent Systems, Heisenbergstrasse 3, D-70569 Stuttgart, Germany \\ ${ }^{3}$ Empa, Swiss Federal Laboratories for Materials Science and Technology, Laboratory for Joining Technologies and Corrosion, \\ Ueberlandstrasse 129, 8600 Duebendorf, Switzerland \\ ${ }^{4}$ Institute of Physics, Chinese Academy of Sciences, Beijing 100190, China \\ ${ }^{5}$ Institute for Materials Science, University of Stuttgart, Heisenbergstrasse 3, D-70569 Stuttgart, Germany
}

(Received 12 August 2016; revised manuscript received 22 February 2017; published 13 March 2017)

\begin{abstract}
This work discloses a surprising, previously unknown heterogeneous growth mode. Namely, large-area, thin sheets of single-crystalline Ge were observed to grow laterally on top of a polycrystalline Al substrate, covering as many as tens of differently oriented Al grains at low temperatures. The observation of the Ge crystal-growth process by in situ heating transmission electron microscopy demonstrates an intriguing type of "faceted" growth: the growth of single-crystalline Ge thin sheets proceeding Al-grain by Al-grain on top of the polycrystalline $\mathrm{Al}$ substrate. The crystalline Ge growth front tends to align along the lines of intersection of the $\mathrm{Al}$ grain boundaries with the Al surface. Such an unusual heterogeneous growth mode has been shown to be a consequence of the strong anisotropy of the energy of the crystalline/crystalline (here: c-Ge/c-Al) interfaces.
\end{abstract}

DOI: 10.1103/PhysRevB.95.094109

\section{INTRODUCTION}

Heterogeneous epitaxial growth of crystalline thin films on crystalline substrates is a phenomenon of crucial importance for components in numerous modern technologies, such as microelectronic and magnetic storage devices, solid-state lasers, and light-emitting diodes (see Ref. [1] and references therein). It is well known that the heterogeneous epitaxial growth is largely controlled by the (mis)match of the lattices of the film and the substrate, based on which different types of film-substrate orientation relationship may occur (e.g., cube-on-cube [2-4], domain epitaxy [5], Bain orientation relationship [6], etc.). Thereby the orientation, the quality, and the properties of the film can be controlled by selection of suitable single-crystal substrates of specific surface orientations $[1,5]$.

In this paper, a surprising, previously unknown heterogeneous growth mode is disclosed. Large-area, thin sheets of single-crystalline $\mathrm{Ge}$ (c-Ge) were observed to grow laterally on the surface of a polycrystalline Al substrate, covering as many as tens of differently oriented $\mathrm{Al}$ grains of the $\mathrm{Al}$ substrate. The investigation of the c-Ge growth process by in situ transmission electron microscopy (TEM) revealed an intriguing type of "faceted" growth: the growth of single-crystalline Ge thin sheets proceeds Al-grain by Al-grain on top of the polycrystalline Al substrate. The c-Ge growth front tends to align along the lines of intersection of $\mathrm{Al}$ grain boundaries (GBs) with the surface of the contacting polycrystalline Al substrate. Such an unusual heterogeneous growth mode was shown to be a consequence of the pronounced anisotropy of the energy of the crystalline/crystalline (here: $\mathrm{c}-\mathrm{Ge} / \mathrm{c}-\mathrm{Al}$ ) interfaces. Thereby a route is opened to the controlled growth of single crystals at low temperatures on polycrystalline substrates.

\footnotetext{
*Corresponding author: z.wang@tju.edu.cn
}

\section{EXPERIMENTAL PROCEDURES}

Specimen preparation was carried out in an ultrahigh vacuum (UHV) molecular beam epitaxy system (base pressure $<2 \times 10^{-10}$ mbar). First, a 50-nm-thick polycrystalline $\mathrm{Al}$ (c-Al) thin film was deposited at room temperature (RT) on a 20-nm thin amorphous $\mathrm{SiO}_{2}$ membrane substrate (SiMPore, Inc.) by thermal evaporation from a pyrolytic boron nitride crucible filled with pure Al (purity $\geqslant 99.95 \mathrm{wt} \%$ ). Then, without breaking the UHV, a 15-nm-thick amorphous Ge (a-Ge) layer was deposited on top of the Al sublayer at RT by thermal evaporation from a graphite crucible filled with pure Ge (purity $\geqslant 99.999 \mathrm{wt} \%$ ). The growth rates of each sublayer were calibrated separately by employing spectroscopic ellipsometry (J.A. Woollam M-2000 ellipsometer) or surface profiling (Veeco Dektak 8 profilometer) measurements of the layer thicknesses for various growth times. The thus-deposited a-Ge/c-Al bilayer specimens were taken out of the growth chamber and directly used for subsequent in situ heating analytic TEM investigations.

The in situ heating energy-filtered TEM (EF-TEM) experiments were carried out in a Zeiss Sub-eV-Sub-Ångstrom Microscope, which is operated at $200 \mathrm{keV}$ and equipped with an electrostatic monochromator and a MANDOLINE filter [7]. The specimen was heated in situ using a Gatan 652 double-tilt heating stage in the microscope in the temperature range of $100{ }^{\circ} \mathrm{C}$ to $150{ }^{\circ} \mathrm{C}$. The temperature of the holder tip was monitored by a calibrated silicon diode mounted on the holder tip, which provides a sensitive temperature response with a precision of $0.5^{\circ} \mathrm{C}$.

\section{RESULTS AND DISCUSSION}

Plan-view energy-filtered TEM images of an as-deposited 15-nm a-Ge/50-nm c-Al specimen are shown in Fig. 1(a), taken at zero electron-energy loss and at electron-energy loss $\Delta E=-15 \mathrm{eV}$ (corresponding to the bulk plasmon absorption of $\mathrm{Al}[8])$. Since the Ge sublayer is amorphous, the 



FIG. 1. (a) Plan-view zero-loss (left-hand side) and energyfiltered (at $\Delta E=-15 \mathrm{eV}$ ) TEM images of an as-deposited 15-nm a-Ge/50-nm c-Al bilayer specimen, showing the columnar grain structure of the polycrystalline Al film. (b) Plan-view zero-loss (left-hand side) and energy-filtered (at $\Delta E=-15 \mathrm{eV}$; right-hand side) TEM images of a 15-nm a-Ge/50-nm c-Al bilayer after in situ heating at $150{ }^{\circ} \mathrm{C}$ for $15 \mathrm{~min}$. In the energy-filtered $(\Delta E=-15 \mathrm{eV})$ TEM image, Al-rich regions appear bright and Ge-rich regions appear dark.

contrast in these TEM images reveals clearly the (columnar) polycrystalline grain structure of the c-Al sublayer. After in situ annealing of the bilayer at $150{ }^{\circ} \mathrm{C}$ for $15 \mathrm{~min}$, crystallization of a-Ge has occurred, as evidenced by the corresponding TEM images shown in Fig. 1(b) (see what follows). The here-observed a-Ge crystallization temperature of $150{ }^{\circ} \mathrm{C}$ is much lower than the bulk crystallization temperature of a-Ge, which is as high as $500^{\circ} \mathrm{C}$ [9]. Such a phenomenon is known as so-called metal-induced crystallization [10,11]. The EF-TEM image at $\Delta E=-15 \mathrm{eV}$ is shown at the right-hand side of Fig. 1(b), in which Al-rich regions appear bright and Ge-rich regions appear dark. The Ge-rich regions are crystalline, as follows from the diffraction contrast observed in the corresponding zero-loss TEM image shown at the left-hand side of Fig. 1(b).

Each separate c-Ge region (c-Ge thin "sheet"), which is about $0.5-1 \mu \mathrm{m}$ large and $20-\mathrm{nm}$ thick, is single crystalline as demonstrated by high-resolution transmission electron microscopy (HRTEM) (see, e.g., Fig. 2, and Figs. S1-S3 of the Supplemental Material [12]). The single-crystalline c-Ge sheets have developed laterally on top of the polycrystalline Al substrate, apparently Al-grain by Al-grain, with curved and "faceted" growth fronts which run more or less along the lines of intersection of the Al GBs with the Al surface [see, e.g., Fig. 1(b)]. The surfaces of some of the Al grains are "bypassed" by the heterogeneously growing c-Ge sheet, leading to the formation of holes in the grown c-Ge sheet.

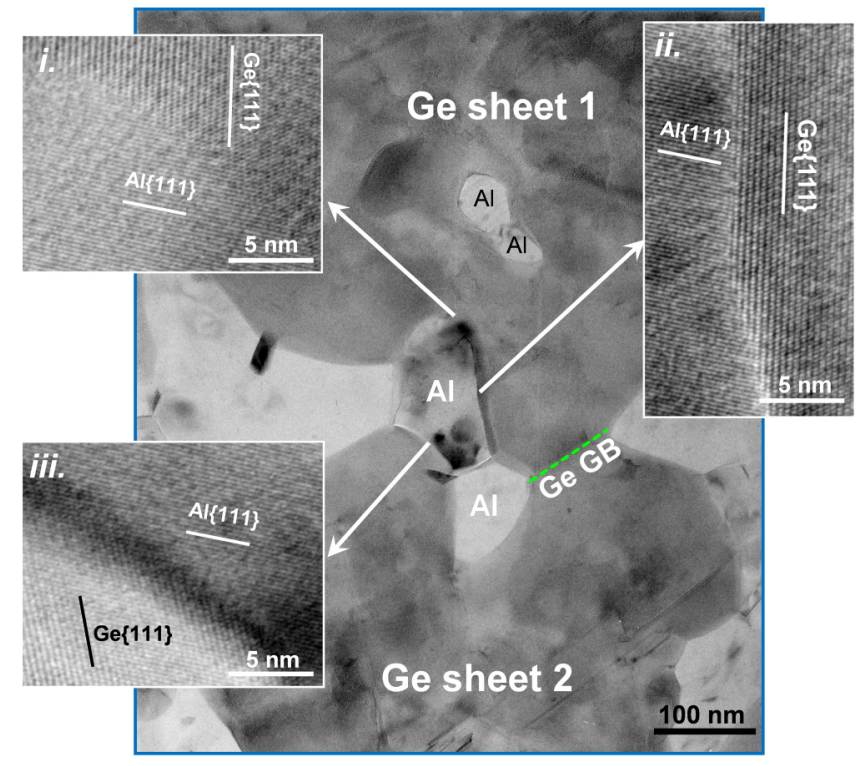

FIG. 2. Plan-view HRTEM analyses of a $15-\mathrm{nm}$ a-Ge/50-nm cAl bilayer after in situ heating at $150{ }^{\circ} \mathrm{C}$ for $15 \mathrm{~min}$. The image shows two representative, separately developed large thin "sheets" of single-crystalline Ge (1\# and 2\#). The lateral growth of the thin c-Ge sheets became stagnant at certain lines of intersection of the Al grain boundaries with the Al surface (see insets $\boldsymbol{i}, \boldsymbol{i} \boldsymbol{i}$, and $\boldsymbol{i i i}$ ).

The "shadows"/presence of many Al grains at (behind) the projected location of the c-Ge thin sheets can be discerned in Fig. 1(b) [see also Figs. 3(a) and 3(b)]. Energy-dispersive $\mathrm{x}$-ray spectroscopy measurements of the same specimens showed that the original a-Ge has largely been depleted (on average $\sim 98$ at. $\% \mathrm{Al}, \sim 2$ at. $\% \mathrm{Ge}$ ) at the projected location outside the c-Ge sheets, whereas $\mathrm{Al}$ and $\mathrm{Ge}$ both occur $(\sim 65$ at. $\% \mathrm{Al}, \sim 35$ at. $\% \mathrm{Ge})$ at the projected location where c-Ge sheets have developed.

A detailed HRTEM analysis of the $\mathrm{c}-\mathrm{Ge}$ thin sheets formed upon in situ annealing of the a-Ge/c-Al specimen at $150^{\circ} \mathrm{C}$ for $15 \mathrm{~min}$ is given in Fig. 2. The representative image shows two separately developed large thin Ge sheets (denoted 1 and 2). Both Ge sheets in the image have been shown to be single crystalline but of different crystal orientation. A Ge GB has formed at the location where the two Ge sheets have come into contact (marked by the green dashed line in Fig. 2). Evidently, the lateral growth of the thin c-Ge sheets has become stagnant at certain lines of intersection of the Al GBs with the surface of the contacting Al substrate, leading to the formation of "faceted" and curved growth fronts of the c-Ge sheets. Two $\mathrm{Al}$ grains can be seen at the (lateral) location between the two c-Ge sheets (see center of Fig. 2). Two small "bypassed" $\mathrm{Al}$ grains are observable within the $\mathrm{c}-\mathrm{Ge}$ sheet 1 .

A detailed EF-TEM analysis of a c-Ge sheet formed upon heating at $150{ }^{\circ} \mathrm{C}$ for $15 \mathrm{~min}$ is presented in Figs. 3(a)-3(c). It confirms indeed that the $\mathrm{c}-\mathrm{Ge}$ sheet is growing on top of the polycrystalline Al substrate Al-grain by Al-grain like [see, in particular, the EF-TEM image at $\Delta E=-15 \mathrm{eV}$ in Fig. 3(b)]. The stagnation of the growth of the $\mathrm{c}-\mathrm{Ge}$ sheet [its growth front is outlined in green in Figs. 3(a) and 3(b)] at the projected locations of many Al GBs is clearly observable. The formed 

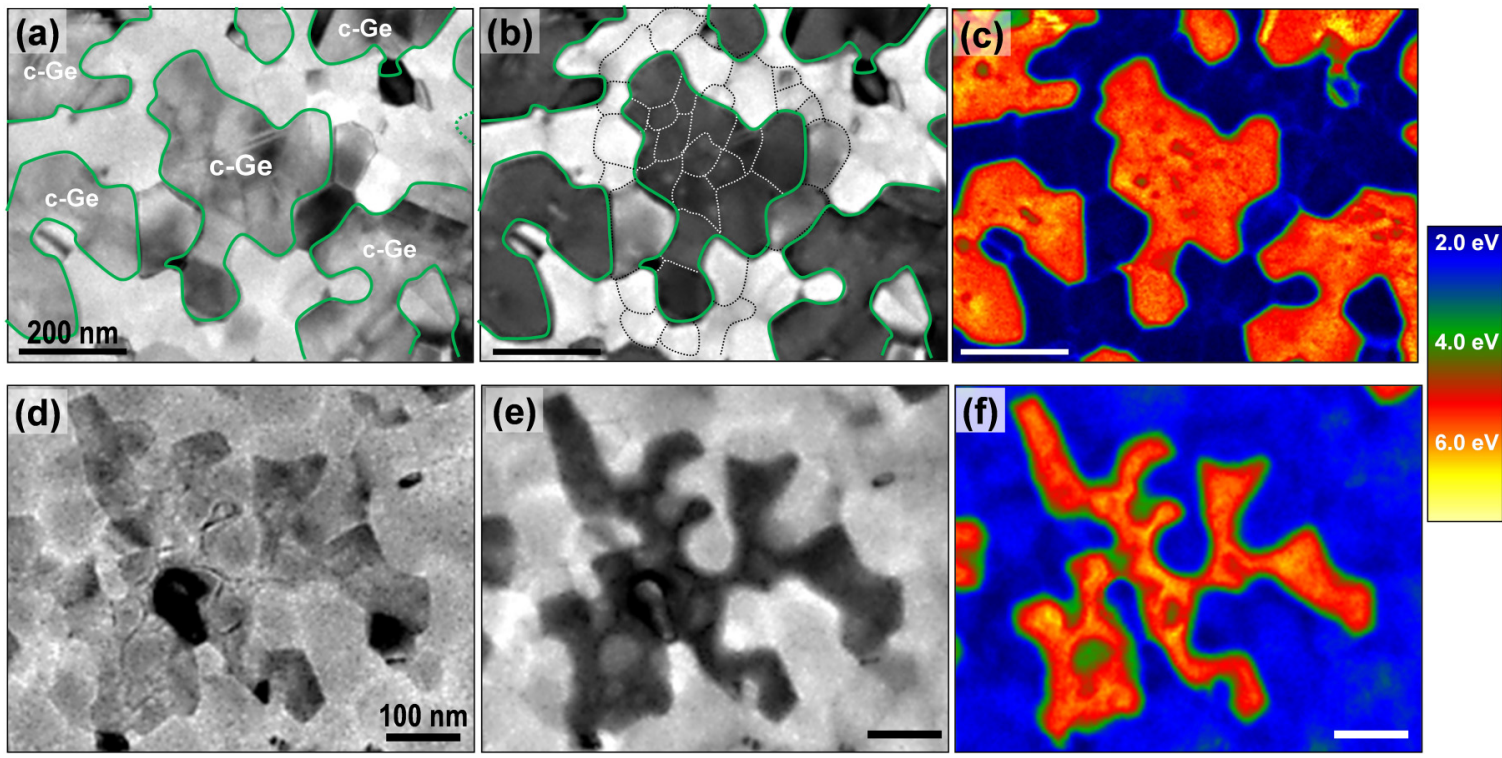

FIG. 3. (a)-(c) Plan-view (a) zero-loss, (b) energy-filtered at $\Delta E=-15 \mathrm{eV}$ TEM images and (c) map of the FWHM of the plasmon-loss peak, taken from a 15-nm a-Ge/50-nm c-Al bilayer after in situ heating at $150{ }^{\circ} \mathrm{C}$ for $15 \mathrm{~min}$. (d), (e) Plan-view (d) zero-loss, (e) energy-filtered at $\Delta E=-15 \mathrm{eV}$ TEM images, and (f) map of the FWHM of the plasmon-loss peak, taken from a $15-\mathrm{nm}$ a-Ge/50-nm c-Al bilayer after in situ heating at $100{ }^{\circ} \mathrm{C}$ for $15 \mathrm{~min}$. Note that the $\mathrm{FWHM}$ of the plasmon-loss peak of $\mathrm{Al}$ is much narrower than that of Ge, and thereby the Ge-rich regions (i.e., the c-Ge sheets) can be very well discerned (in red) in the FWHM mappings in (c) and (f).

c-Ge sheet shown in the center of Fig. 3(b) has covered more than ten $\mathrm{Al}$ grains.

This intriguing heterogeneous growth mode has also been observed at an even lower temperature of only $100^{\circ} \mathrm{C}$. The EF-TEM analysis of a $15-\mathrm{nm}$ a-Ge/50-nm c-Al specimen annealed at $100^{\circ} \mathrm{C}$ for $15 \mathrm{~min}$ is shown in Figs. 3(d)-3(f). Similar to the c-Ge sheets formed at $150{ }^{\circ} \mathrm{C}$, the c-Ge sheets formed at $100^{\circ} \mathrm{C}$ appear to grow also Al-grain by Al-grain, and thus show a "faceted" grain front. As compared to the results at $150{ }^{\circ} \mathrm{C}$, the c-Ge sheet morphology at $100^{\circ} \mathrm{C}$ is more branchlike [compare Figs. 3(d)-3(f) and Figs. 3(a)-3(c)]. This may indicate that, at the lower temperature, less $\mathrm{Al}$ grains are "active" (i.e., associated with a relatively low energy barrier which can be overcome; see discussion below) for the Al-induced crystallization of a-Ge.

The observed heterogeneous growth mode (i.e., growth of single crystals on polycrystals) has not been reported before. The mechanism for such an unusual crystal-growth mode is discussed in the following; see Fig. 4.

The first step of the heterogeneous growth is the nucleation of a Ge crystallite on top of an $\mathrm{Al}$ grain [grain 0, Fig. 4(a)]. This interface-mediated nucleation of $\mathrm{c}-\mathrm{Ge}$ has been shown to be possible to occur at the a-Ge/c-Al interface at very low temperatures $\left(\geqslant 50^{\circ} \mathrm{C}\right)$ on the basis of interface thermodynamic calculation $[9,13]$. The critical thickness above which the bulk Gibbs energy overcompensates the increase of the interface energies upon crystallization is smaller than the thickness of a-Ge adjacent to the metal of weakened covalent bonds and thus of increased atomic mobility [14]. Then, as shown in Fig. 4(b), the c-Ge may grow laterally towards and on top of the surface of the $\mathrm{Al}$ grains (grains 1-7) surrounding grain 0 . The crystallization of a-Ge will be retarded by a relatively high interface energy of the formed c-Ge/c-Al interface due to crystallization of a-Ge $\left(\gamma_{\langle\mathrm{Ge}\rangle\langle\langle\mathrm{Al}\rangle}\right)$ [15], with an average value of about $0.35 \mathrm{~J} \mathrm{~m}^{-2}$ at $100{ }^{\circ} \mathrm{C}$ [13], as compared to that of the original a-Ge/c-Al interface $\left(\gamma_{\{\mathrm{Ge}\} \mid\langle\mathrm{Al}\rangle}\right)[15], \sim 0.05 \mathrm{~J} \mathrm{~m}^{-2}$ at $100^{\circ} \mathrm{C}$ [13]. Depending on the interface energy of c-Ge with different $\mathrm{Al}$ grains $\left(\gamma_{\langle\mathrm{Ge}\rangle}^{i} \mid\langle\mathrm{Al}\rangle, i=1-7\right)$, the growth of c-Ge proceeds preferentially across the $\mathrm{Al}$ grains associated with lower interface energies $\gamma_{\langle\mathrm{Ge}\rangle \mid\langle\mathrm{Al}\rangle}^{i}$ (in this drawing, grains 2, 4, and 5) and becomes stagnant at the Al GBs towards Al grains associated with relatively high $\gamma_{\langle\mathrm{Ge}\rangle \mid\langle\mathrm{Al}\rangle}^{i}$ [in this drawing, grains 1, 3, 6, and 7; see also Fig. 4(d) for a schematic cross-sectional view]. Subsequently, as shown in Fig. 4(c), the c-Ge continues to grow, now from grains 2, 4, and 5, towards the surface of adjacent $\mathrm{Al}$ grains associated with relatively low $\gamma_{\langle\mathrm{Ge}\rangle\langle\langle\mathrm{Al}\rangle}^{i}$, thus forming a faceted thin sheet morphology of c-Ge as observed in Figs. 2 and 3. Since $\gamma_{\langle\mathrm{Ge}\rangle\langle\langle\mathrm{Al}\rangle}^{i}$ increases with decreasing temperatures [13], at relatively low temperatures only few (say, one or two) of the adjacent $\mathrm{Al}$ grains could be energetically favorable enough for the growth of the c-Ge sheet above. This would lead to the formation of more branch-shaped c-Ge, as observed in Figs. 3(d)-3(f) $\left(T=100^{\circ} \mathrm{C}\right)$. At higher temperatures, more (say, three to five) of the adjacent $\mathrm{Al}$ grains could energetically accept to be covered with c-Ge, i.e., allow the growth of the c-Ge sheet on top. This would lead to the formation of more compacted c-Ge sheets, as observed in Figs. 2 and 3(a)-3(c) $\left(T=150^{\circ} \mathrm{C}\right)$.

After growth stagnation of $\mathrm{c}-\mathrm{Ge}$ at the intersection of an $\mathrm{Al} \mathrm{GB}$ with the $\mathrm{Al}$ surface, it is energetically more preferred that the $\mathrm{Ge}$ atoms of the a-Ge on the adjacent $\mathrm{Al}$ grain [grain 1 in the drawing of Figs. 4(d) and 4(e)] now diffuse along the a-Ge/c-Al interface [14] towards the c-Ge on grain 0 , causing growth of the $\mathrm{c}-\mathrm{Ge}$ sheet on grain 0 perpendicular to the surface (i.e., thickening) and depletion of a-Ge on Al grain 1 , as experimentally observed (Figs. 1 and 3). 
(a)

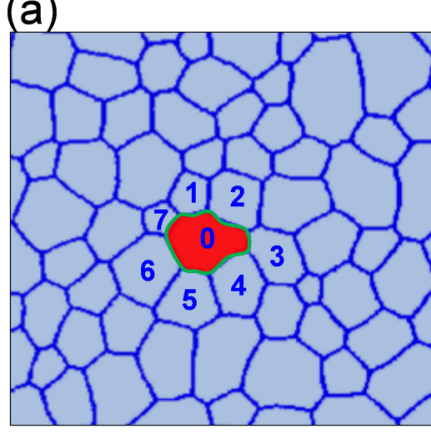

(d) (b)

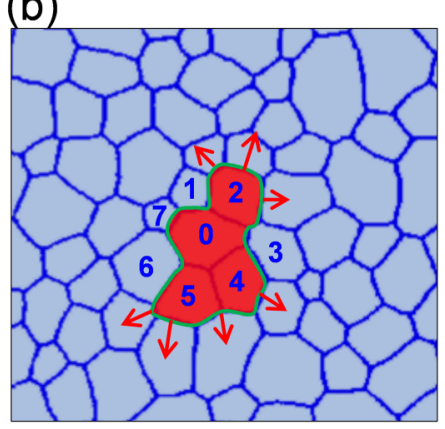

(c)

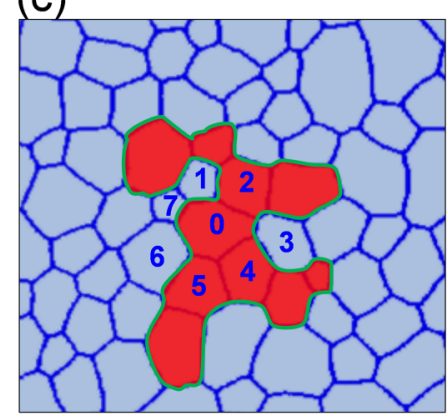

(e)
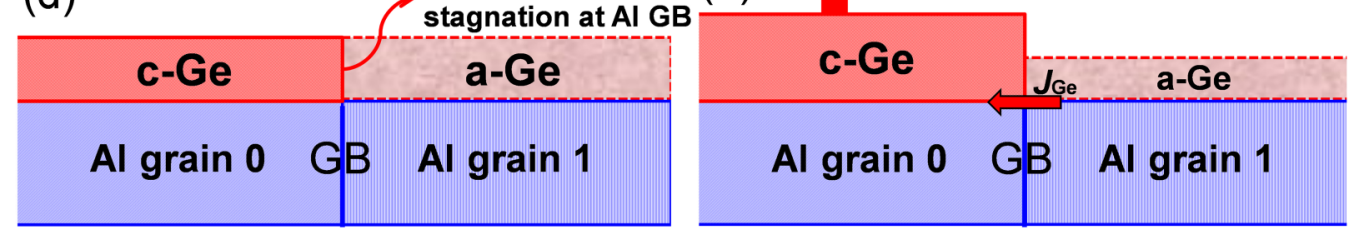

FIG. 4. Schematic illustration of the growth mechanism of a single-crystalline sheet of $\mathrm{Ge}$ (red) on top of a polycrystalline Al substrate, (a)-(c) a top view, (d), (e) a cross-sectional view: (a) first c-Ge is nucleated locally on top of an Al grain (grain 0); (b) the c-Ge grows towards (and on top of) the surface of the $\mathrm{Al}$ grains (grains 1-7) surrounding grain 0. Depending on the interface energy of c-Ge on different $\mathrm{Al}$ grains $\left(\gamma_{\langle\mathrm{Ge}\rangle\langle\mathrm{A} \mid\rangle}^{i}\right)$, the growth of c-Ge proceeds preferentially across the Al grains with lower $\gamma_{\langle\mathrm{Ge}\rangle\langle\langle\mathrm{Al}\rangle}^{i}$ (in this drawing, grains 2, 4, and 5), and becomes stagnant at the $\mathrm{Al} \mathrm{GBs} \mathrm{towards} \mathrm{Al}$ grains with very high $\gamma_{\langle\mathrm{Ge}\rangle \mid\langle\mathrm{Al}\rangle}^{i}$ (in this drawing, grains 1, 3, 6, and 7). (c) The c-Ge continues to grow, now from grains 2, 4, and 5, towards the surface of adjacent Al grains with relatively low $\gamma_{\langle\mathrm{Ge}\rangle\langle\langle\mathrm{Al}\rangle}^{i}$, thus forming a faceted thin sheet morphology of c-Ge, as, e.g., observed in Fig. 3. (d) A cross-sectional view: the growth front of c-Ge reaches the location of intersection of an Al GB with the $\mathrm{Al}$ surface. If the interface energy of $\mathrm{c}-\mathrm{Ge}$ on the adjacent $\mathrm{Al}$ grain (grain 1), $\gamma_{\langle\mathrm{Ge}\rangle\langle\langle\mathrm{Al}\rangle}^{1}$, is relatively high, the growth of c-Ge will become stagnant at the $\mathrm{Al} \mathrm{GB}$ between grains 0 and 1. (e) Upon the c-Ge growth stagnation at the $\mathrm{Al} \mathrm{GB,} \mathrm{Ge} \mathrm{atoms} \mathrm{in} \mathrm{the} \mathrm{a-Ge} \mathrm{layer} \mathrm{on} \mathrm{top} \mathrm{of} \mathrm{Al} \mathrm{grain}$ 1 will diffuse along the a-Ge/Al interface towards the $\mathrm{c}-\mathrm{Ge}$ sheet on $\mathrm{Al}$ grain 0 , thus leading to the growth of a c-Ge sheet perpendicular to the surface and the depletion of a-Ge on Al grain 1.

A key point of the above growth mechanism is the anisotropy of the energy of the crystalline/crystalline interface, which involves that for a single-crystalline Ge sheet (i.e., with a fixed crystal orientation), the $\mathrm{c}-\mathrm{Ge} / \mathrm{c}-\mathrm{Al}$ interface energy is much dependent on the orientation of the underlying c-Al. The orientations of the (c-Al) grains in the polycrystalline (Al) film grown on an amorphous substrate have been known to be close to random [16]. Already more or less straightforward considerations make clear that the surface energy of a crystalline solid is significantly anisotropic, with relatively low surface energy for the more close-packed surfaces [17]. However, such treatments become much more complex considering the interface energy of a crystalline/crystalline interface [18-22].

According to Ref. [23], the $\langle A\rangle \mid\langle B\rangle$ interface energy is given by

$$
\gamma_{\langle A\rangle \mid\langle B\rangle}=\gamma_{\langle A\rangle \mid\langle B\rangle}^{\text {mis }}+\gamma_{\langle A\rangle \mid\langle B\rangle}^{\text {chem }},
$$

where the mismatch energy term $\gamma_{\langle A\rangle \mid\langle B\rangle}^{\mathrm{mis}}$ originates from the structural mismatch between the lattices of $\langle A\rangle$ and $\langle B\rangle$, and the chemical energy term $\gamma_{\langle A\rangle \mid\langle B\rangle}^{\text {chem }}$ originates from the chemical interaction of materials $\langle A\rangle$ and $\langle B\rangle$.

If $A$ and $B$ are the same material, the $\gamma_{\langle A\rangle \mid\langle A\rangle}^{\text {chem }}$ term is zero, and it follows for the grain-boundary energy $\gamma_{\langle A\rangle}^{\mathrm{GB}}$,

$$
\gamma_{\langle A\rangle}^{\mathrm{GB}} \equiv \gamma_{\langle A\rangle \mid\langle A\rangle}=\gamma_{\langle A\rangle \mid\langle A\rangle}^{\mathrm{mis}} .
$$

The chemical interaction energy term $\gamma_{\langle A\rangle \mid\langle B\rangle}^{\text {chem }}$ depends on the atomic density at the interface plane [23] and should therefore be anisotropic.
The mismatch term $\gamma_{\langle A\rangle \mid\langle B\rangle}^{\mathrm{mis}}$ is often discussed in literature to be quasi-isotropic for a "general" (i.e., except coincident-sitelattice interfaces) high-angle solid-solid interfaces (including GBs), with an average value of [23]

$$
\gamma_{\langle A\rangle \mid\langle B\rangle}^{\mathrm{mis}}=\frac{1}{6}\left(\gamma_{\langle A\rangle}^{\mathrm{S}}+\gamma_{\langle B\rangle}^{\mathrm{S}}\right)
$$

and thus

$$
\gamma_{\langle A\rangle}^{\mathrm{GB}}=\frac{1}{3} \gamma_{\langle A\rangle}^{\mathrm{S}} .
$$

Recent theoretical work $[19,20]$ has, however, indicated the occurrence of significant GB energy anisotropy in facecentered-cubic and body-centered-cubic metals: the GB energy $\gamma_{\langle A\rangle}^{\mathrm{GB}}$ depends strongly on the misorientation angle and the GB plane orientation, with an average value close to that predicted according to Eq. (4). Analogously, the $\gamma_{\langle A\rangle \mid\langle B\rangle}^{\mathrm{mis}}$ should also be strongly anisotropic.

As a result of the above considerations, it must be expected that both $\gamma_{\langle A\rangle \mid\langle B\rangle}^{\text {mis }}$ and $\gamma_{\langle A\rangle \mid\langle B\rangle}^{\text {chem }}$, and hence $\gamma_{\langle A\rangle \mid\langle B\rangle}$, are (strongly) anisotropic. Indeed, using the methods described in Refs. [23,24], it is calculated that a semicoherent $\mathrm{Al}(111) / \mathrm{Ge}(111)$ interface has an energy of about $0.24 \mathrm{~J} \mathrm{~m}^{-2}$, which is significantly smaller than the average energy of about $0.35 \mathrm{~J} \mathrm{~m}^{-2}$ for "general" $\langle\mathrm{Al}\rangle /\langle\mathrm{Ge}\rangle$ interfaces [13].

Reversely, the heterogeneous growth mode observed in this project provides strong experimental proof for the occurrence of crystalline/crystalline $\langle A\rangle /\langle B\rangle$ interface energy anisotropy. 


\section{CONCLUSIONS}

An unusual heterogeneous crystal-growth mode is disclosed by which relatively large single-crystalline thin sheets can be grown on top of polycrystals. The growth of the single-crystalline thin sheets proceeds laterally on top of the polycrystalline substrate, in a substrate-grain-by-grain manner. Such a growth mode is controlled fundamentally by the anisotropy of the crystalline/crystalline interface energies. This growth mode may enable the development of single- crystalline devices at low temperatures on polycrystalline substrates.

\section{ACKNOWLEDGMENTS}

Z.W. acknowledges support by the Thousand Talents Program for Distinguished Young Scholars of China and by the National Natural Science Foundation of China (Grant No. 51571148). The in situ heating TEM experiments were carried out at the Stuttgart Center for Electron Microscopy.
[1] H. Brune, in Surface and Interface Science (Wiley-VCH Verlag $\mathrm{GmbH} \& \mathrm{Co}, \mathrm{KGaA}$, Weinheim, Germany, 2013), p. 421.

[2] F. C. Frank and J. H. van der Merwe, Proc. R. Soc. Lond. A 198, 216 (1949).

[3] J. W. Matthews and A. E. Blakeslee, J. Cryst. Growth 27, 118 (1974).

[4] E. A. Fitzgerald, Mater. Sci. Rep. 7, 87 (1991).

[5] J. Narayan and B. C. Larson, J. Appl. Phys. 93, 278 (2003).

[6] J. Buschbeck, I. Opahle, M. Richter, U. K. Rößler, P. Klaer, M. Kallmayer, H. J. Elmers, G. Jakob, L. Schultz, and S. Fähler, Phys. Rev. Lett. 103, 216101 (2009).

[7] C. T. Koch, W. Sigle, R. Höschen, M. Rühle, E. Essers, G. Benner, and M. Matijevic, Microsc. Microanal. 12, 506 (2006).

[8] R. F. Egerton, Electron Energy-Loss Spectroscopy in the Electron Microscope, 2nd ed. (Plenum Press, New York, 1996).

[9] Z. M. Wang, L. P. H. Jeurgens, J. Y. Wang, and E. J. Mittemeijer, Adv. Eng. Mater. 11, 131 (2009).

[10] Z. M. Wang, J. Y. Wang, L. P. H. Jeurgens, and E. J. Mittemeijer, Phys. Rev. Lett. 100, 125503 (2008).

[11] Metal-Induced Crystallization: Fundamentals and Applications, edited by Z. M. Wang, L. P. H. Jeurgens, and E. J. Mittemeijer (Pan Stanford, Singapore, 2015).

[12] See Supplemental Material at http://link.aps.org/supplemental/ 10.1103/PhysRevB.95.094109 for plan-view bright-field/darkfield TEM and HRTEM characterization of the c-Ge nanosheets.
[13] Z. M. Wang, J. Y. Wang, L. P. H. Jeurgens, and E. J. Mittemeijer, Phys. Rev. B 77, 045424 (2008).

[14] Z. M. Wang, L. P. H. Jeurgens, W. Sigle, and E. J. Mittemeijer, Phys. Rev. Lett. 115, 016102 (2015).

[15] In this paper, " \langle\rangle " denotes the crystalline phase whereas " \{\} " denotes the amorphous phase.

[16] X-ray diffraction pole-figure measurements performed in this project have shown that the deposited Al film has a weak (111) texture with fully random in-plane orientations.

[17] I. Galanakis, N. Papanikolaou, and P. H. Dederichs, Surf. Sci. 511, 1 (2002).

[18] A. P. Sutton and R. W. Balluffi, Interfaces in Crystalline Materials (Oxford University Press, Oxford, UK, 1995).

[19] D. L. Olmsted, S. M. Foiles, and E. A. Holm, Acta Mater. 57, 3694 (2009).

[20] G. S. Rohrer, J. Mater. Sci. 46, 5881 (2011).

[21] V. V. Bulatov, B. W. Reed, and M. Kumar, Acta Mater. 65, 161 (2014).

[22] P. R. M. van Beers, V. G. Kouznetsova, M. G. D. Geers, M. A. Tschopp, and D. L. McDowell, Acta Mater. 82, 513 (2015).

[23] L. P. H. Jeurgens, Z. M. Wang, and E. J. Mittemeijer, Int. J. Mater. Res. 100, 1281 (2009).

[24] F. Reichel, L. P. H. Jeurgens, and E. J. Mittemeijer, Phys. Rev. B 74, 144103 (2006). 


\title{
Heterogeneous growth of single crystals on polycrystals
}

\author{
Zumin Wang, Lars P. H. Jeurgens, Lin Gu, Eric J. Mittemeijer
}
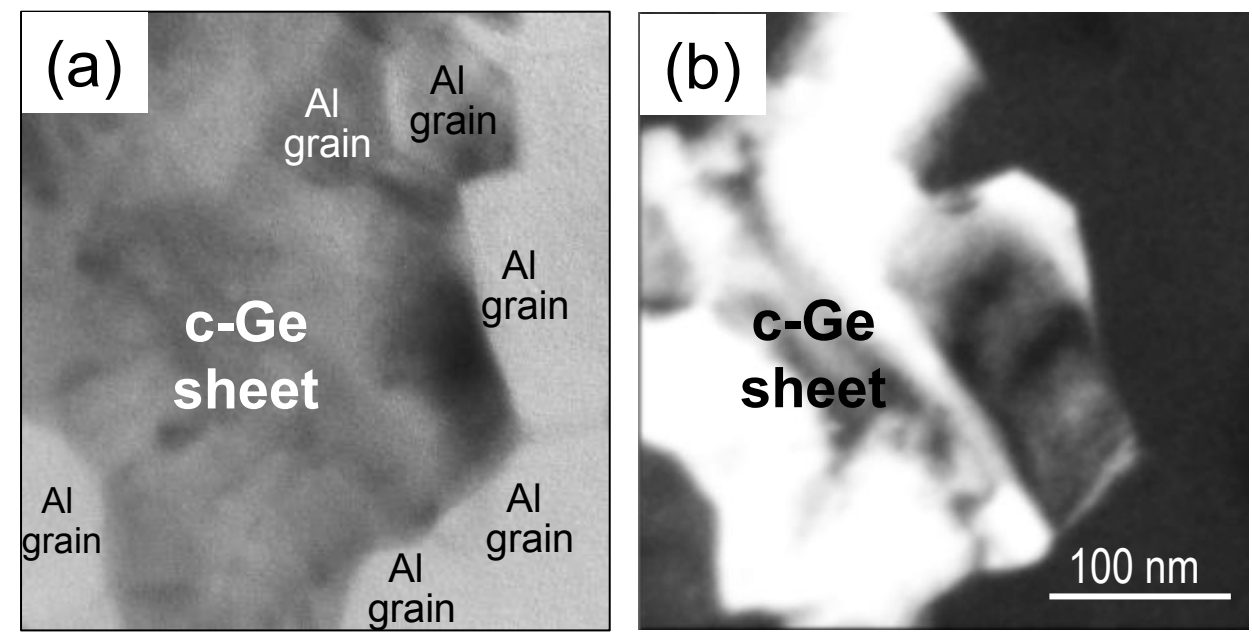

Figure S1. Plan-view (a) bright-field and (b) corresponding dark-field TEM images of a 15-nm a-Ge/50-nm c-Al bilayer after in situ heating at $150{ }^{\circ} \mathrm{C}$ for $15 \mathrm{~min}$. The dark-field image has been taken by positioning the objective aperture on a diffraction spot corresponding to c-Ge. The dark-field TEM has confirmed the single-crystalline structure of a representative, large c-Ge sheet developed on top of polycrystalline Al. 


\section{Al grain}

\section{Al grain}

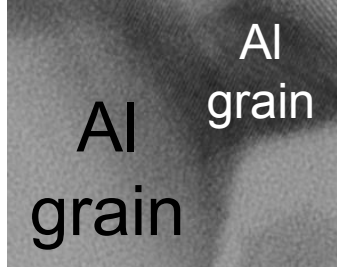

\section{Al grain}

\section{$20 \mathrm{~nm}$}

Figure S2. Plan-view HRTEM image of a 15-nm a-Ge/50-nm c-Al bilayer after in situ heating at $150{ }^{\circ} \mathrm{C}$ for $15 \mathrm{~min}$. The image confirms the single-crystalline structure of a large Ge sheet developed on top of polycrystalline $\mathrm{Al}$. 


\section{$\mathrm{C}-\mathrm{Ge}$ sheet}

\section{Al grain}

\section{Al grain}

Figure S3. Another plan-view HRTEM image of a 15-nm a-Ge/50-nm c-Al bilayer after

in situ heating at $150{ }^{\circ} \mathrm{C}$ for $15 \mathrm{~min}$. The image confirms the single-crystalline structure of a large Ge sheet developed on top of polycrystalline Al. 